\title{
Surface energy of the one-dimensional supersymmetric $t-J$ model with unparallel boundary fields
}

\author{
Fakai Wen,,$^{a, b, c}$ Zhan-Ying Yang, ${ }^{b, d, 1}$ Tao Yang, ${ }^{a, b, d}$ Kun Hao, ${ }^{a, b}$ Junpeng Cao ${ }^{c, e, f, 1}$ and \\ Wen-Li Yang ${ }^{a, b, d, 1}$ \\ ${ }^{a}$ Institute of Modern Physics, Northwest University, \\ 229 Taibai Beilu, Xi'an 710069, China \\ ${ }^{b}$ Shaanxi Key Laboratory for Theoretical Physics Frontiers, \\ 229 Taibai Beilu, Xi'an 710069, China \\ ${ }^{c}$ Institute of Physics, Chinese Academy of Sciences, \\ 8 3rd South Street, Zhongguancun, Beijing 100190, China \\ ${ }^{d}$ School of Physics, Northwest University, \\ 229 Taibai Beilu, Xi'an 710069, China \\ e School of Physical Sciences, University of Chinese Academy of Sciences, \\ Beijing, China \\ ${ }^{f}$ Collaborative Innovation Center of Quantum Matter, \\ Beijing, China \\ E-mail: fakaiwen@126.com, zyyang@nwu.edu.cn, yangt@nwu.edu.cn, \\ haoke72@163.com, junpengcao@iphy.ac.cn, wlyang@nwu.edu.cn
}

ABstract: We investigate the thermodynamic limit of the exact solution, which is given by an inhomogeneous $T-Q$ relation, of the one-dimensional supersymmetric $t-J$ model with unparallel boundary magnetic fields. It is shown that the contribution of the inhomogeneous term at the ground state satisfies the $L^{-1}$ scaling law, where $L$ is the system-size. This fact enables us to calculate the surface (or boundary) energy of the system. The method used in this paper can be generalized to study the thermodynamic limit and surface energy of other models related to rational R-matrices.

KeYwords: Bethe Ansatz, Lattice Integrable Models

ARXIV EPRINT: 1712.04199

\footnotetext{
${ }^{1}$ Corresponding author.
} 


\section{Contents}

1 Introduction $\quad 1$

2 Bethe ansatz solutions $\quad 2$

3 Finite-size effects $\quad 6$

3.1 Region of $\xi<0$ and $\xi^{\prime} \leq 1 / 2 \quad 8$

3.2 Region of $\xi<0$ and $1 / 2<\xi^{\prime}<1 \quad 9$

4 Surface energy 10

4.1 Region of $\xi<0$ and $\xi^{\prime} \leq 1 / 2 \quad 10$

4.2 Region of $\xi<0$ and $1 / 2<\xi^{\prime}<1 \quad 12$

5 Conclusions 13

\section{Introduction}

The $t-J$ model is the strongly repulsive limit of the well-known Hubbard model [1-3], which has played a fundamental and important role in strongly correlated electron systems. The model is also one of the cornerstone models in the study of high- $T_{c}$ superconductivity [47]. In general, the Hamiltonian includes nearest-neighbor hopping $(t)$ and nearest-neighbor spin exchange and charge interactions $(J)$ (see below (1.1)) for the periodic case [8]. For the open case, the Hamiltonian also includes the boundary chemical potentials $\chi_{1}, \chi_{L}$ and the boundary fields $\mathbf{h}_{1}, \mathbf{h}_{L}[9,10]$, i.e.,

$$
\begin{aligned}
H= & -t \sum_{\alpha, j=1}^{L-1} \mathcal{P}\left[c_{j, \alpha}^{\dagger} c_{j+1, \alpha}+c_{j+1, \alpha}^{\dagger} c_{j, \alpha}\right] \mathcal{P}+J \sum_{j=1}^{L-1}\left[\mathbf{S}_{j} \cdot \mathbf{S}_{j+1}-\frac{1}{4} n_{j} n_{j+1}\right] \\
& +\chi_{1} n_{1}+2 \mathbf{h}_{1} \cdot \mathbf{S}_{1}+\chi_{L} n_{L}+2 \mathbf{h}_{L} \cdot \mathbf{S}_{L}
\end{aligned}
$$

where $L$ is the total number of lattice sites and the coupling constants $\chi_{1}, \chi_{L}$ and $\mathbf{h}_{1}, \mathbf{h}_{L}$ are given by (2.17) below. The operators $c_{j, \alpha}$ and $c_{j, \alpha}^{\dagger}$ are the annihilation and creation operators of the electron with spin $\alpha= \pm 1$ on the lattice site $j$, which satisfies anticommutation relations, i.e., $\left\{c_{i, \alpha}^{\dagger}, c_{j, \tau}\right\}=\delta_{i, j} \delta_{\alpha, \tau}$. There are only three possible states at the lattice site $i$ due to the factor $\mathcal{P}=\left(1-n_{j,-\alpha}\right)$ projects out double occupancies. The number operator on the site $j$ is $n_{j}=\sum_{\alpha= \pm 1} n_{j, \alpha}$ with $n_{j, \alpha}=c_{j, \alpha}^{\dagger} c_{j, \alpha}$, and the total number operator is $\hat{N}=\sum_{j=1}^{L} n_{j}$. The spin operators $S=\sum_{j=1}^{L} S_{j}, \quad S^{\dagger}=\sum_{j=1}^{L} S_{j}^{\dagger}$ and $S^{z}=\sum_{j=1}^{L} S_{j}^{z}$ with $S_{j}=c_{j, 1}^{\dagger} c_{j,-1}, S_{j}^{\dagger}=c_{j,-1}^{\dagger} c_{j, 1}$ and $S_{j}^{z}=\frac{1}{2}\left(n_{j, 1}-n_{j,-1}\right)$ being the local operators form the $s u(2)$ algebra. 
At the supersymmetric points $J= \pm 2 t$, the Hamiltonian in one spatial dimension is supersymmetric and integrable [11-18]. One can obtain the exact solution of the onedimensional supersymmetric $t-J$ model with periodic boundary condition or parallel boundary fields by the nested Bethe ansatz method $[8,9]$ or the off-shell Bethe ansatz [1921]. Based on the exact solution, the properties of the $t-J$ models, for example, surface energy, the elementary excitations, the correlation functions and the thermodynamics have attracted a great attention [22-25]. Compared with the periodic case and parallel boundary fields case, the one-dimensional supersymmetric $t-J$ model with unparallel boundary fields is the most general integrable case. Due to the U(1)-symmetry-broken for the case with unparallel boundary fields, the conventional methods have failed to solve the model for a long time [26]. The exact solution of the one-dimensional supersymmetric $t-J$ model with unparallel boundary fields $[27,28]$ was obtained recently, which will enable one further to study the thermodynamic limit and surface energy [29-31] of the model.

In this paper, we will study the thermodynamic limit and boundary effects of the supersymmetric $t-J$ model with unparallel boundary fields. Based on the former work [32], one can not employ the thermodynamic Bethe ansatz (TBA) method directly to approach the thermodynamic limit of the $t-J$ model due to the inhomogeneous term in the $T-Q$ relation. Therefore, the first thing should be addressed is the contribution of the inhomogeneous term. Without losing generality, we take the region of $\chi_{1}>1$ and $\chi_{L}<1$ as an example. Through the analysis of finite-lattice systems, it is shown that the contribution of the inhomogeneous term in the associated $T-Q$ relation to the ground state energy satisfies the scaling law $L^{-1}$, where $L$ is the system-size. Based on this fact, by using the standard thermodynamic Bethe ansatz method, we find that all the Bethe roots are real at the ground state in the region of $\chi_{1}>1$ and $0 \leq \chi_{L}<1$. While in the region of $\chi_{1}>1$ and $\chi_{L}<0$, besides the real Bethe roots, there exists a pure imaginary root which corresponds to a stable boundary bound state. Furthermore, the surface energy of the system is calculated. Comparison of the surface energy from the analytic expressions with that from the Hamiltonian by the extrapolation method, we show that they coincide with each other very well.

The plan of the paper is as follows. We briefly review the Bethe ansatz solutions of the one-dimensional supersymmetric $t-J$ model with unparallel boundary fields in section 2 . In section 3 , we focus on the contribution of the inhomogeneous term to the ground state energy. In section 4, with the help of the Bethe ansatz solution for the finite-size system, we study the thermodynamic limit and surface energy of the model. We summarize our results and give some discussions in section 5 .

\section{Bethe ansatz solutions}

In this paper we consider the system with $J=2 t$ and $t=-1$, which corresponds to the supersymmetric and integrable case [8]. Let $\mathbf{V}^{(\mathbf{m} \mid \mathbf{n})}=\mathbf{V}^{\mathbf{m}} \oplus \mathbf{V}^{\mathbf{n}}$ denotes a graded linear space with an orthonormal basis $\{|i\rangle, i=1, \cdots, m+n\}$ having the Grassmann parity (denoted by $\epsilon_{i}$ ): $\epsilon_{i}=0$ for $i=1, \cdots, m$ and $\epsilon_{i}=1$ for $i=m+1, \cdots, m+n$, which endows the fundamental representation of $s u(m \mid n)$ algebra [33]. For the supersymmetric $t-J$ model, we have $m=1$ and $n=2$ [8]. The integrability of the model is associated 
with the $R$-matrix

$$
R_{i, j}(u)=u+\eta \Pi_{i, j},
$$

where $u$ is the spectral parameter and $\eta$ is the crossing parameter, and $\Pi_{i, j}$ is the $Z_{2}$-graded permutation operator

$$
\left(\Pi_{i, j}\right)_{a_{i} a_{j}}^{b_{i} b_{j}}=\delta_{a_{i} b_{j}} \delta_{a_{j} b_{i}}(-1)^{\epsilon_{b_{i}} \epsilon_{b_{j}}}
$$

The $R$-matrix satisfies the graded Yang-Baxter equation

$$
R_{12}(u-v) R_{13}(u) R_{23}(v)=R_{23}(v) R_{13}(u) R_{12}(u-v),
$$

and possesses the properties:

$$
\begin{array}{rlrl}
\text { Initial condition: } & R_{12}(0) & =\eta \Pi_{12}, \\
\text { Unitarity relation: } & R_{12}(u) R_{21}(-u) & =\rho_{1}(u) \times \mathrm{id}, \\
\text { Crossing } & \text { Unitarity relation: } & R_{12}^{s t_{1}}(-u+\eta) R_{21}^{s t_{1}}(u) & =\rho_{2}(u) \times \mathrm{id},
\end{array}
$$

where $\rho_{1}(u)=-(u-\eta)(u+\eta), \rho_{2}(u)=-u(u-\eta), R_{21}(u)=\Pi_{12} R_{21}(u) \Pi_{12}$ and $s t_{i}$ denotes the super transposition in the $i$-th space $\left(A^{s t}\right)_{i j}=A_{j i}(-1)^{\epsilon_{i}\left[\epsilon_{i}+\epsilon_{j}\right]}$. Here and below we adopt the standard notation: for any matrix $A \in \operatorname{End}\left(\mathbf{V}^{(\mathbf{m} \mid \mathbf{n})}\right), A_{j}$ is a super embedding operator in the graded tensor product space $\mathbf{V}^{(\mathbf{m} \mid \mathbf{n})} \otimes \mathbf{V}^{(\mathbf{m} \mid \mathbf{n})} \otimes \cdots$, which acts as $A$ on the $j$-th space and as an identity on the other factor spaces; $R_{i j}(u)$ is a super embedding operator of $R$-matrix in the graded tensor product space, which acts as an identity on the factor spaces except for the $i$-th and $j$-th ones.

In this paper we consider the most general reflection matrices: ${ }^{1}$

$$
K^{-}(u)=\left(\begin{array}{ccc}
\xi+u & 0 & 0 \\
0 & \xi+c u & 2 c_{1} u \\
0 & 2 c_{2} u & \xi-c u
\end{array}\right)
$$

which satisfy the reflection equation (RE)

$$
R_{12}(u-v) K_{1}^{-}(u) R_{21}(u+v) K_{2}^{-}(v)=K_{2}^{-}(v) R_{12}(u+v) K_{1}^{-}(u) R_{21}(u-v),
$$

and

$$
K^{+}(u)=\left(\begin{array}{ccc}
\xi^{\prime}-u & 0 & 0 \\
0 & \xi^{\prime}-\frac{\eta}{2}+c^{\prime}\left(-u+\frac{\eta}{2}\right) & 2 c_{1}^{\prime}\left(-u+\frac{\eta}{2}\right) \\
0 & 2 c_{2}^{\prime}\left(-u+\frac{\eta}{2}\right) & \xi^{\prime}-\frac{\eta}{2}-c^{\prime}\left(-u+\frac{\eta}{2}\right)
\end{array}\right),
$$

which satisfies the dual RE respectively

$$
\begin{aligned}
R_{12}(u-v) K_{1}^{+}(v) & R_{21}(\eta-u-v) K_{2}^{+}(u) \\
& =K_{2}^{+}(u) R_{12}(\eta-u-v) K_{1}^{+}(v) R_{21}(u-v) .
\end{aligned}
$$

\footnotetext{
${ }^{1}$ Without losing the generalization, the $K^{ \pm}(u)$ given by $(2.7)$ and (2.9) are the most general $K$-matrices of the model and satisfy $\left[K^{-}(u), K^{+}(v)\right] \neq 0$. This fact gives rise to that they cannot be diagonalized simultaneously (which corresponds to the non-diagonal (or unparallel) boundary fields), and that there does not exist an obvious reference state on which the conventional Bethe ansatz [26] can be performed.
} 
The above parameters in (2.7) and (2.9) have to satisfy the restrictions [28]

$$
c^{2}+4 c_{1} c_{2}-1=0, \quad c^{\prime 2}+4 c_{1}^{\prime} c_{2}^{\prime}-1=0,
$$

to make sure that the associated $K$-matrices satisfy the RE (2.8) and its dual (2.10).

Let us introduce the one-row monodromy matrices

$$
\begin{aligned}
& T_{0}(u)=R_{0 L}(u) R_{0 L-1}(u) \cdots R_{01}(u), \\
& \hat{T}_{0}(u)=R_{1,0}(u) \cdots R_{L-1,0}(u) R_{L, 0}(u),
\end{aligned}
$$

and the double-row monodromy matrix

$$
\mathcal{U}(u)=T(u) K^{-}(u) \hat{T}(u) .
$$

Then the transfer matrix is given by

$$
t(u)=\operatorname{str}_{0}\left\{K_{0}^{+}(u) \mathcal{U}_{0}(u)\right\},
$$

where $\operatorname{str}_{0}$ denotes the supertrace carried out in the auxiliary space $[8,9]$.

With the same procedure introduced in [11], one can show that $[t(u), t(v)]=0$, which ensures the integrability of the model described by Hamiltonian (1.1). The first order derivative of the logarithm of the transfer matrix $t(u)$ yields Hamiltonian (1.1)

$$
H=\left.\frac{\eta}{2} \frac{d \ln t(u)}{d u}\right|_{u=0}-\frac{\eta}{2 \xi}+\frac{\eta-2 \xi^{\prime}}{2\left(\eta-\xi^{\prime}\right)}+2 \hat{N}-L+1,
$$

where the coupling constants in the Hamiltonian are expressed in terms of the parameters in the corresponding $K$-matrices given in (2.7), (2.9) and (2.11) as follows:

$$
\begin{aligned}
\chi_{1} & =1-\frac{\eta}{2 \xi}, & h_{1}^{x} & =\frac{\eta}{2 \xi}\left(c_{2}+c_{1}\right), \quad h_{1}^{y}=\frac{\eta}{2 \xi}\left(c_{2}-c_{1}\right) i, \quad h_{1}^{z}=-\frac{\eta}{2 \xi} c, \\
\chi_{L} & =1-\frac{\eta}{2\left(\eta-\xi^{\prime}\right)}, & h_{L}^{x} & =\frac{\eta}{2\left(\eta-\xi^{\prime}\right)}\left(c_{2}^{\prime}+c_{1}^{\prime}\right), \\
h_{L}^{y} & =\frac{\eta}{2\left(\eta-\xi^{\prime}\right)}\left(c_{2}^{\prime}-c_{1}^{\prime}\right) i, & h_{L}^{z} & =-\frac{\eta}{2\left(\eta-\xi^{\prime}\right)} c^{\prime} .
\end{aligned}
$$

It is remarked that the total number operator $\hat{N}$ is still a conserved charge for the model described by Hamiltonian (1.1), i.e., $[H, \hat{N}]=0$.

By combining the algebraic Bethe ansatz and the off-diagonal Bethe ansatz [28], the eigenvalues $\Lambda(u)$ of the transfer matrix $t(u)$ is given by an inhomogeneous $T-Q$ relation

$$
\begin{aligned}
\Lambda(u)= & w_{3}(u)(\xi+u)(u+\eta)^{2 L} \frac{Q(u-\eta)}{Q(u)}-u^{2 L} \bar{a}(u) \frac{Q(u-\eta) Q^{(1)}(u+\eta)}{Q(u) Q^{(1)}(u)} \\
& -u^{2 L} \bar{d}(u) \frac{Q^{(1)}(u-\eta)}{Q^{(1)}(u)}+2 h u^{2 L+1}\left(u-\frac{\eta}{2}\right) \frac{Q(u-\eta)}{Q^{(1)}(u)},
\end{aligned}
$$

where

$$
\begin{aligned}
w_{3}(u) & =\xi^{\prime}-u-\frac{\eta}{2 u+\eta}\left(2 \xi^{\prime}-\eta\right), & h & =1+\left(c c^{\prime}+2 c_{1} c_{2}^{\prime}+2 c_{1}^{\prime} c_{2}\right), \\
\bar{a}(u) & =\frac{u-\frac{\eta}{2}}{u+\frac{\eta}{2}}\left(u+\xi^{\prime}\right)(u+\xi), & \bar{d}(u) & =\left(u-\xi^{\prime}\right)(u-\xi), \\
Q^{(1)}(u) & =\prod_{l=1}^{M}\left(u-\lambda_{l}\right)\left(u+\lambda_{l}\right), & Q(u) & =\prod_{k=1}^{M}\left(u-v_{k}\right)\left(u+v_{k}+\eta\right) .
\end{aligned}
$$


For simplicity, we introduce two new parameters $\theta$ and $\varphi$ which satisfy

$$
c=\cos (\theta), \quad c_{1}=\frac{\sin (\theta)}{2} e^{i \varphi}, \quad c_{2}=\frac{\sin (\theta)}{2} e^{-i \varphi},
$$

and other two new parameters $\theta^{\prime}$ and $\varphi^{\prime}$ which satisfy

$$
c^{\prime}=-\varepsilon \cos \left(\theta^{\prime}\right), \quad c_{1}^{\prime}=-\varepsilon \frac{\sin \left(\theta^{\prime}\right)}{2} e^{i \varphi^{\prime}}, \quad c_{2}^{\prime}=-\varepsilon \frac{\sin \left(\theta^{\prime}\right)}{2} e^{-i \varphi^{\prime}},
$$

where

$$
\varepsilon=\operatorname{sgn}\left(\mathbf{h}_{1} \cdot \mathbf{h}_{L}\right)=\frac{\mathbf{h}_{1} \cdot \mathbf{h}_{L}}{\left|\mathbf{h}_{1} \cdot \mathbf{h}_{L}\right|} .
$$

The above parameterizations make the constraints (2.11) fulfilled automatically. We further assume the parameters $\eta, \xi, \theta, \phi, \xi^{\prime}, \theta^{\prime}, \phi^{\prime}$ being real numbers to ensure the hermitian of Hamiltonian (1.1). For $\varepsilon=1$ case, the possible values of the parameters $\xi$ and $\xi^{\prime}$ are constrained to be taken in the region of $\xi<0$ and $\xi^{\prime}<1$ or $\xi>0$ and $\xi^{\prime}>1$, respectively. While for $\varepsilon=-1$ case, they are taken in the region of $\xi<0$ and $\xi^{\prime}>1$ or $\xi>0$ and $\xi^{\prime}<1$, respectively. In this paper, we choose the region of $\xi<0$ and $\xi^{\prime}<1$ as an example. It is straightforward to extend the analysis below to other ranges of the fields. ${ }^{2}$

Using the relations (2.19)-(2.21), we obtain

$$
h=1-\varepsilon\left[\cos (\theta) \cos \left(\theta^{\prime}\right)+\sin (\theta) \sin \left(\theta^{\prime}\right) \cos \left(\varphi-\varphi^{\prime}\right)\right] .
$$

It should be remarked that if the two boundary fields $\mathbf{h}_{1}$ and $\mathbf{h}_{L}$ are parallel (i.e., $\theta^{\prime}=\theta$, $\varphi^{\prime}=\varphi$ ) or anti-parallel (i.e., $\theta^{\prime}+\theta=\pi,\left|\varphi^{\prime}-\varphi\right|=\pi$ ), the associated $K^{ \pm}$-matrices can be diagonalized simultaneously. In this case, the $\mathrm{U}(1)$ symmetry in the spin sector is recovered and the constant $h$ given by (2.23) vanishes.

To ensure $\Lambda(u)$ to be a polynomial, the residues of $\Lambda(u)$ at the poles $v_{j}$ and $\lambda_{j}$ must vanish, i.e., the $2 M$ parameters $\left\{v_{j} \mid j=1, \cdots, M\right\}$ and $\left\{\lambda_{j} \mid j=1, \cdots, M\right\}$ must satisfy the nested Bethe ansatz equations (BAEs)

$$
\left(\xi^{\prime}-v_{j}-\frac{\eta}{2 v_{j}+\eta}\left(2 \xi^{\prime}-\eta\right)\right)\left(\xi+v_{j}\right)\left(v_{j}+\eta\right)^{2 L}=v_{j}^{2 L} \bar{a}\left(v_{j}\right) \frac{Q^{(1)}\left(v_{j}+\eta\right)}{Q^{(1)}\left(v_{j}\right)}, j=1, \cdots, M,
$$

and

$$
\begin{aligned}
\bar{a}\left(\lambda_{j}\right) Q\left(\lambda_{j}-\eta\right) Q^{(1)}\left(\lambda_{j}+\eta\right) & +\bar{d}\left(\lambda_{j}\right) Q\left(\lambda_{j}\right) Q^{(1)}\left(\lambda_{j}-\eta\right) \\
& =2 h \lambda_{j}\left(\lambda_{j}-\frac{\eta}{2}\right) Q\left(\lambda_{j}\right) Q\left(\lambda_{j}-\eta\right), \quad j=1, \cdots, M
\end{aligned}
$$

From the relation (2.16), we have the eigenvalue of Hamiltonian (1.1) in terms of the Bethe roots, which is given by

$$
\begin{aligned}
E & =\left.\frac{\eta}{2} \frac{d \ln \Lambda(u)}{d u}\right|_{u=0}-\frac{\eta}{2 \xi}+\frac{\eta-2 \xi^{\prime}}{2\left(\eta-\xi^{\prime}\right)}+2 M-L+1 \\
& =\sum_{k=1}^{M} \frac{\eta^{2}}{v_{k}\left(v_{k}+\eta\right)}+2 M .
\end{aligned}
$$

\footnotetext{
${ }^{2}$ We note that the conclusions may not change in the other ranges of the fields.
} 


\begin{tabular}{|rccc|c|c|}
\hline$v_{1}$ & $v_{2}$ & $\lambda_{1}$ & $\lambda_{2}$ & $E_{n}$ & $n$ \\
\hline$-0.5000-0.2786 i$ & - & $0.4756+0.0000 i$ & - & -1.052578 & 1 \\
- & - & - & - & 0 & 2 \\
$-0.5000-0.5801 i$ & - & $1.5481-0.0000 i$ & - & 0.295101 & 3 \\
$-0.5000+0.2731 i$ & $-0.5000+1.6511 i$ & $-0.4393-0.0000 i$ & $0.0000+1.6139 i$ & 0.583040 & 4 \\
$-0.5000+1.9038 i$ & - & $0.0000+2.1272 i$ & - & 1.741892 & 5 \\
$-0.0015+0.3109 i$ & $-0.0015-0.3109 i$ & $-0.0000+0.3403 i$ & $2.1227-0.0000 i$ & 2.141851 & 6 \\
$-0.5000-0.4606 i$ & $0.5419-0.0000 i$ & $0.9406+0.0000 i$ & $2.1803-0.0000 i$ & 3.033107 & 7 \\
$0.4957-0.0000 i$ & - & $1.6356+0.0000 i$ & - & 3.348918 & 8 \\
$0.4798-0.0000 i$ & $-0.5000-1.3235 i$ & $-0.0000+1.5362 i$ & $2.0858+0.0000 i$ & 4.908668 & 9 \\
\hline
\end{tabular}

Table 1. Solutions of BAEs (2.24)-(2.25) for the case of $L=2, \eta=1, \xi=0.6, \theta=\pi / 5, \phi=$ $\pi / 3, \xi^{\prime}=1.5, \theta^{\prime}=2 \pi / 3, \phi^{\prime}=\pi / 4$. The symbol $n$ indicates the number of the eigenvalues, and $E_{n}$ is the corresponding eigenenergy. The energy $E_{n}$ calculated from (2.26) is the same as that from the exact diagonalization of Hamiltonian (1.1).

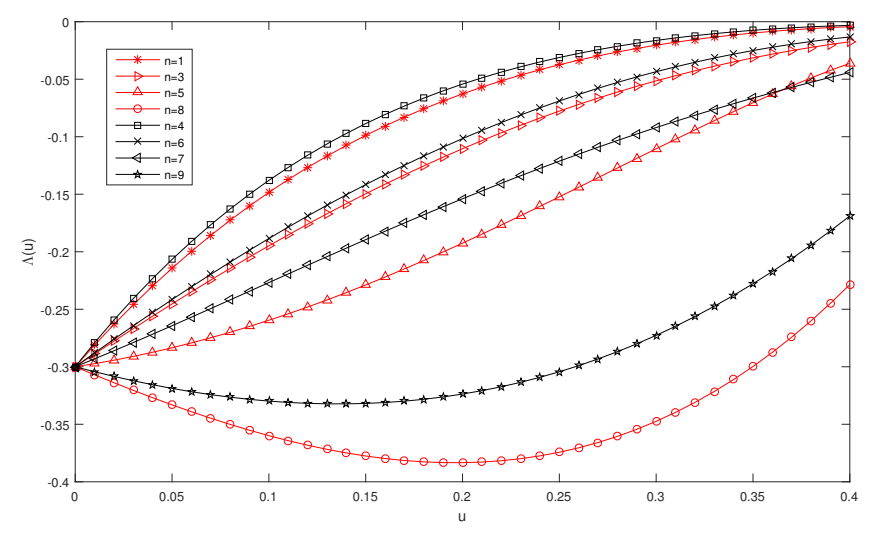

Figure 1. (color online) $\Lambda(u)$ vs. $u$ for the case of $L=2$. The curves calculated from $T-Q$ relation (2.18) and the nested BAEs (2.24)-(2.25) are exactly the same as those obtained from the exact diagonalization of the transfer matrix $t(u)$.

The numerical solutions of BAEs (2.24)-(2.25) and the corresponding eigenvalues of Hamiltonian (1.1) for $L=2$ is shown in table 1, while the calculated $\Lambda(u)$ curves for $L=2$ are shown in figure 1. Those numerical simulations imply that the inhomogeneous $T-Q$ relation (2.18) and the BAEs (2.24)-(2.25) indeed give the correct and complete spectrum of the one-dimensional supersymmetric $t-J$ model with unparallel boundary fields $[26,34,35]$.

\section{$3 \quad$ Finite-size effects}

In order to study the contribution of the inhomogeneous term (the last term in (2.18)) to the ground state energy, we first consider the $T-Q$ relation without the inhomogeneous 
term, ${ }^{3}$ i.e.,

$$
\begin{aligned}
\Lambda_{\mathrm{hom}}(u)= & w_{3}(u)(\xi+u)(u+\eta)^{2 L} \frac{Q(u-\eta)}{Q(u)}-u^{2 L} \bar{a}(u) \frac{Q(u-\eta) Q^{(1)}(u+\eta)}{Q(u) Q^{(1)}(u)} \\
& -u^{2 L} \bar{d}(u) \frac{Q^{(1)}(u-\eta)}{Q^{(1)}(u)} .
\end{aligned}
$$

The singular property of the $T-Q$ relation (3.1) gives rise to the associated BAEs

$$
\frac{\mu_{j}+\left(\frac{\eta}{2}-\xi^{\prime}\right)}{\mu_{j}-\left(\frac{\eta}{2}-\xi^{\prime}\right)}\left(\frac{\mu_{j}+\frac{\eta}{2}}{\mu_{j}-\frac{\eta}{2}}\right)^{2 L}=-\prod_{l=1}^{\bar{M}} \frac{\mu_{j}-\lambda_{l}+\frac{\eta}{2}}{\mu_{j}-\lambda_{l}-\frac{\eta}{2}} \frac{\mu_{j}+\lambda_{l}+\frac{\eta}{2}}{\mu_{j}+\lambda_{l}-\frac{\eta}{2}}
$$

and

$$
\begin{aligned}
\frac{\lambda_{j}-\frac{\eta}{2}}{\lambda_{j}+} \frac{\frac{\eta}{2}}{\left(\lambda_{j}+\xi^{\prime}\right)\left(\lambda_{j}+\xi\right)} & \\
& =-\prod_{j=1}^{M} \frac{\left(\lambda_{j}-\mu_{k}+\frac{\eta}{2}\right)\left(\lambda_{j}+\mu_{k}+\frac{\eta}{2}\right)}{\left(\lambda_{j}-\mu_{k}-\frac{\eta}{2}\right)\left(\lambda_{j}+\mu_{k}-\frac{\eta}{2}\right)} \prod_{l=1}^{\bar{M}} \frac{\left(\lambda_{j}-\lambda_{l}-\eta\right)\left(\lambda_{j}+\lambda_{l}-\eta\right)}{\left(\lambda_{j}-\lambda_{l}+\eta\right)\left(\lambda_{j}+\lambda_{l}+\eta\right)},
\end{aligned}
$$

where we have put $v_{j}=\mu_{j}-\frac{\eta}{2}$.

Assume that $\mu_{j} \rightarrow \mu_{j} i, \lambda_{j} \rightarrow \lambda_{j} i$ and $\eta=1$, we obtain

$$
\frac{\mu_{j}-\left(\frac{1}{2}-\xi^{\prime}\right) i}{\mu_{j}+\left(\frac{1}{2}-\xi^{\prime}\right) i}\left(\frac{\mu_{j}-\frac{i}{2}}{\mu_{j}+\frac{i}{2}}\right)^{2 L}=-\prod_{l=1}^{\bar{M}} \frac{\mu_{j}-\lambda_{l}-\frac{i}{2}}{\mu_{j}-\lambda_{l}+\frac{i}{2}} \frac{\mu_{j}+\lambda_{l}-\frac{i}{2}}{\mu_{j}+\lambda_{l}+\frac{i}{2}},
$$

and

$$
\begin{aligned}
& \frac{\lambda_{j}+\frac{i}{2}}{\lambda_{j}-\frac{i}{2}} \frac{\left(\lambda_{j}-\xi^{\prime} i\right)\left(\lambda_{j}-\xi i\right)}{\left(\lambda_{j}+\xi^{\prime} i\right)\left(\lambda_{j}+\xi i\right)} \\
& \quad=-\prod_{k=1}^{M} \frac{\left(\lambda_{j}-\mu_{k}-\frac{i}{2}\right)\left(\lambda_{j}+\mu_{k}-\frac{i}{2}\right)}{\left(\lambda_{j}-\mu_{k}+\frac{i}{2}\right)\left(\lambda_{j}+\mu_{k}+\frac{i}{2}\right)} \prod_{l=1}^{\bar{M}} \frac{\left(\lambda_{j}-\lambda_{l}+i\right)\left(\lambda_{j}+\lambda_{l}+i\right)}{\left(\lambda_{j}-\lambda_{l}-i\right)\left(\lambda_{j}+\lambda_{l}-i\right)} .
\end{aligned}
$$

The corresponding eigenvalue, denoted by $E_{\text {hom }}$, reads

$$
\begin{aligned}
E_{\mathrm{hom}} & =\left.\frac{\eta}{2} \frac{d \ln \Lambda_{\mathrm{hom}}(u)}{d u}\right|_{u=0}-\frac{\eta}{2 \xi}+\frac{\eta-2 \xi^{\prime}}{2\left(\eta-\xi^{\prime}\right)}+2 M-L+1 \\
& =-\sum_{k=1}^{M} \frac{1}{\mu_{k}^{2}+\frac{1}{4}}+2 M .
\end{aligned}
$$

Now, we consider the contribution of the inhomogeneous term in eq. (2.18) to the ground state energy of the system. In order to this, we should analyze the distribution of Bethe roots in the BAEs (3.4) and (3.5). For $\xi<0$ and $\xi^{\prime}<1$ (equivalent to $\chi_{1}>1$ and $\chi_{L}<1$ ), by using the standard thermodynamic Bethe ansatz method and taking the limit

\footnotetext{
${ }^{3}$ It should be emphasized that, for a finite $L, \Lambda_{\text {hom }}(u)$ is different from the exact eigenvalue $\Lambda(u)$ given by (2.18).
} 
of temperature tending to zero, we find that all the Bethe roots are real at the ground state in the region of $\xi<0$ and $\xi^{\prime} \leq 1 / 2$ (equivalent to $\chi_{1}>1$ and $0 \leq \chi_{L}<1$ ). While in region of $\xi<0$ and $1 / 2<\xi^{\prime}<1$ (equivalent to $\chi_{1}>1$ and $\chi_{L}<0$ ), besides the real Bethe roots, there exists an imaginary Bethe root which corresponds to a boundary bound state. We shall discuss them separately.

\subsection{Region of $\xi<0$ and $\xi^{\prime} \leq 1 / 2$}

Firstly, we consider the case of $\xi<0$ and $\xi^{\prime} \leq 1 / 2[23,26]$, in which all the Bethe roots are real at the ground state. Taking the logarithm of BAEs (3.4)-(3.5), we obtain

$$
\begin{aligned}
2 \pi I_{j}= & 2 \arctan \left(\frac{2 \mu_{j}}{1-2 \xi^{\prime}}\right)+4 L \arctan \left(2 \mu_{j}\right) \\
& -\sum_{l=1}^{\bar{M}} 2 \arctan \left(2\left(\mu_{j}-\lambda_{l}\right)\right)+2 \arctan \left(2\left(\mu_{j}+\lambda_{l}\right)\right), \\
2 \pi J_{j}= & 2 \arctan \left(2 \lambda_{j}\right)-2 \arctan \left(\frac{\lambda_{j}}{\xi^{\prime}}\right)-2 \arctan \left(\frac{\lambda_{j}}{\xi}\right) \\
& +\sum_{k=1}^{M} 2 \arctan \left(2\left(\lambda_{j}-\mu_{k}\right)\right)+2 \arctan \left(2\left(\lambda_{j}+\mu_{k}\right)\right) \\
& -\sum_{l=1}^{\bar{M}} 2 \arctan \left(\lambda_{j}-\lambda_{l}\right)+2 \arctan \left(\lambda_{j}+\lambda_{l}\right),
\end{aligned}
$$

where $I_{j}$ and $J_{j}$ are both quantum numbers which determine the eigenenergy and the corresponding eigenstates. It is well-known that the size of the system $L$, with either even or odd value, gives the same physical properties in the thermodynamic limit. Therefore, for simplicity, we set $L$ as an even number.

We define the contribution of the inhomogeneous term to the ground state energy as

$$
E_{\text {inh }}=E_{\text {hom }}-E_{\text {true }}
$$

Here $E_{\text {hom }}$ is the energy of the supersymmetric $t-J$ model calculated by the eigenvalue (3.6) and the BAEs (3.7)-(3.8). $E_{\text {true }}$ is the energy of Hamiltonian (1.1), which can be obtained by using the density matrix renormalization group (DMRG) [36]. For the ground state, the number of Bethe roots reduces to $M=L / 2$ and $\bar{M}=0$,

$$
2 \pi I_{j}=2 \arctan \left(\frac{\mu_{j}}{\zeta}\right)+4 L \arctan \left(2 \mu_{j}\right), \quad j=1, \cdots, M,
$$

where $I_{j} \in\{1,2, \cdots, L / 2\}, \zeta=1 / 2-\xi^{\prime}$ and $\zeta \geq 0$. Then "ground state energy" $E_{\text {hom }}$ is given by equation (3.6) with the constraint (3.10).

The values of $E_{\mathrm{inh}}$, the contribution of the inhomogeneous term to the ground state energy, versus the system size $L$ are shown in figure 2 . From the fitting, we find the power law relation between $E_{\text {inh }}$ and $L$, i.e., $E_{\text {inh }}=\gamma L^{\beta}$. Due to the fact that $\beta \approx-1$, the value of $E_{\text {inh }}$ tends to zero when the size of the system tends to infinity, which means that the 

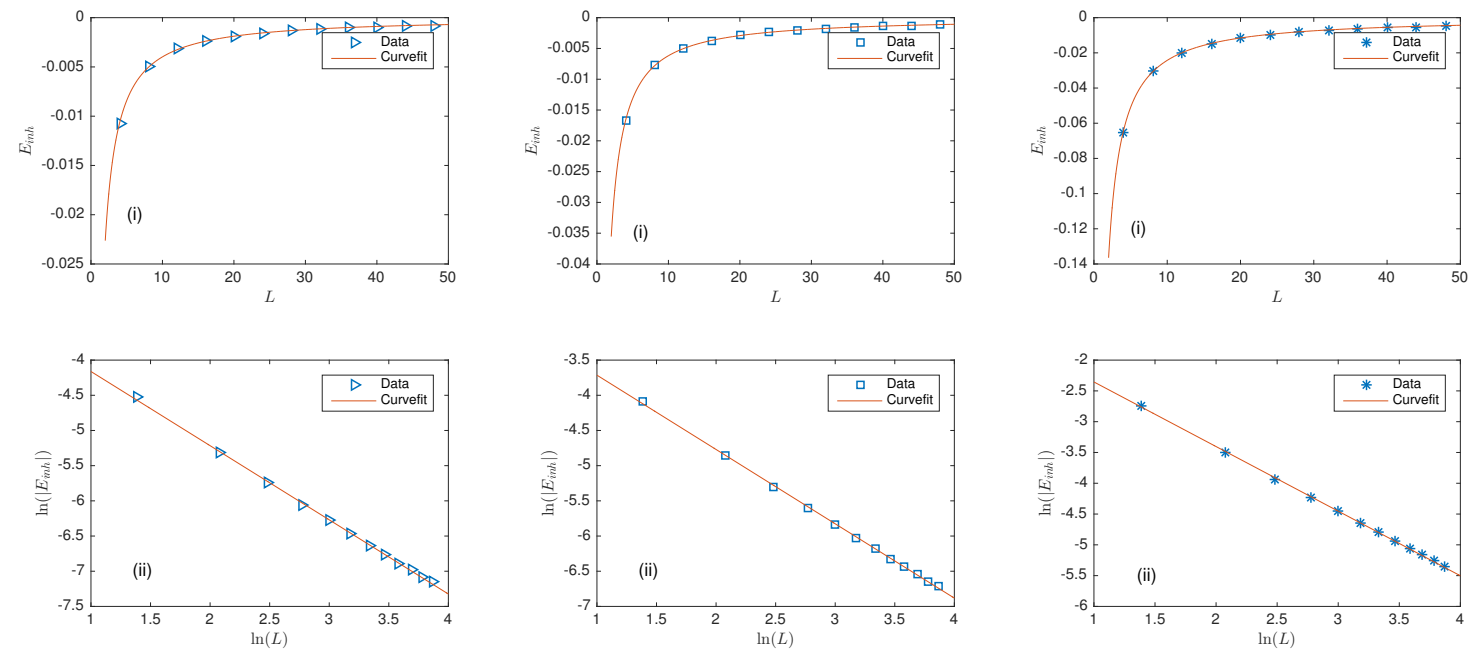

(a) $\theta=\pi / 5$.

(b) $\theta=\pi / 4$.

(c) $\theta=\pi / 2$.

Figure 2. The contribution of the inhomogeneous term to the ground state energy $E_{\text {inh }}$ versus the system size $L$. The data can be fitted as $E_{\text {inh }}=\gamma L^{\beta}\left[\right.$ or $\left.\ln \left(\left|E_{\text {inh }}\right|\right)=p \ln (L)+q\right]$. Here $\xi=-0.1$, $\zeta=0.05, \theta^{\prime}=0, \phi=\phi^{\prime}=0$, (a) $\theta=\pi / 5, \gamma=-0.0478$ and $\beta=-1.080 ;$ (b) $\theta=\pi / 4, \gamma=-0.0755$ and $\beta=-1.087$; (c) $\theta=\pi / 2, \gamma=-0.2871$ and $\beta=-1.073$. Due to the fact $\beta<0$ [or $p<0$ ], when the $L$ tends to infinity, the contribution of the inhomogeneous term tends to zero.

inhomogeneous term in the $T-Q$ relation (2.18) can be neglected in the thermodynamic limit $L, N, M \rightarrow \infty$ with fixed $N / L$ and $M / L$. Therefore, the two boundaries are decoupled from each other completely in the thermodynamic limit. When $h=0$, the unparallel boundary fields degenerate into the parallel one. At this point, the contribution of the inhomogeneous term to the ground state energy $E_{\text {inh }}$ is 0 .

\subsection{Region of $\xi<0$ and $1 / 2<\xi^{\prime}<1$}

In the region of $\xi<0$ and $1 / 2<\xi^{\prime}<1$, one of the Bethe roots at the ground state goes to $\left(\frac{1}{2}-\xi^{\prime}\right) i$ when the system-size $L$ tends to infinity $[26,37-39]$. We note the value of this Bethe root is related with the boundary parameter $\xi^{\prime}$. Without losing generality, we assume that $\mu_{M}=\left(\frac{1}{2}-\xi^{\prime}\right) i+\mathcal{O}\left(e^{-\delta L}\right)=\zeta i+\mathcal{O}\left(e^{-\delta L}\right)$ where $M=L / 2$ and $\delta$ is a small positive number to account for the finite size deviations. This Bethe root contributes a negative bare energy if $-1 / 2<\zeta<0$. The remaining Bethe roots should take real values and satisfy the following BAEs

$$
\frac{\mu_{j}-\zeta i}{\mu_{j}+\zeta i}\left(\frac{\mu_{j}-\frac{i}{2}}{\mu_{j}+\frac{i}{2}}\right)^{2 L}=-1, \quad j=1,2, \cdots, M-1 .
$$

Taking the logarithm of eq. (3.11), we have

$$
2 \pi I_{j}=2 \arctan \left(\frac{\mu_{j}}{\zeta}\right)+4 L \arctan \left(2 \mu_{j}\right), \quad j=1, \cdots, M-1,
$$



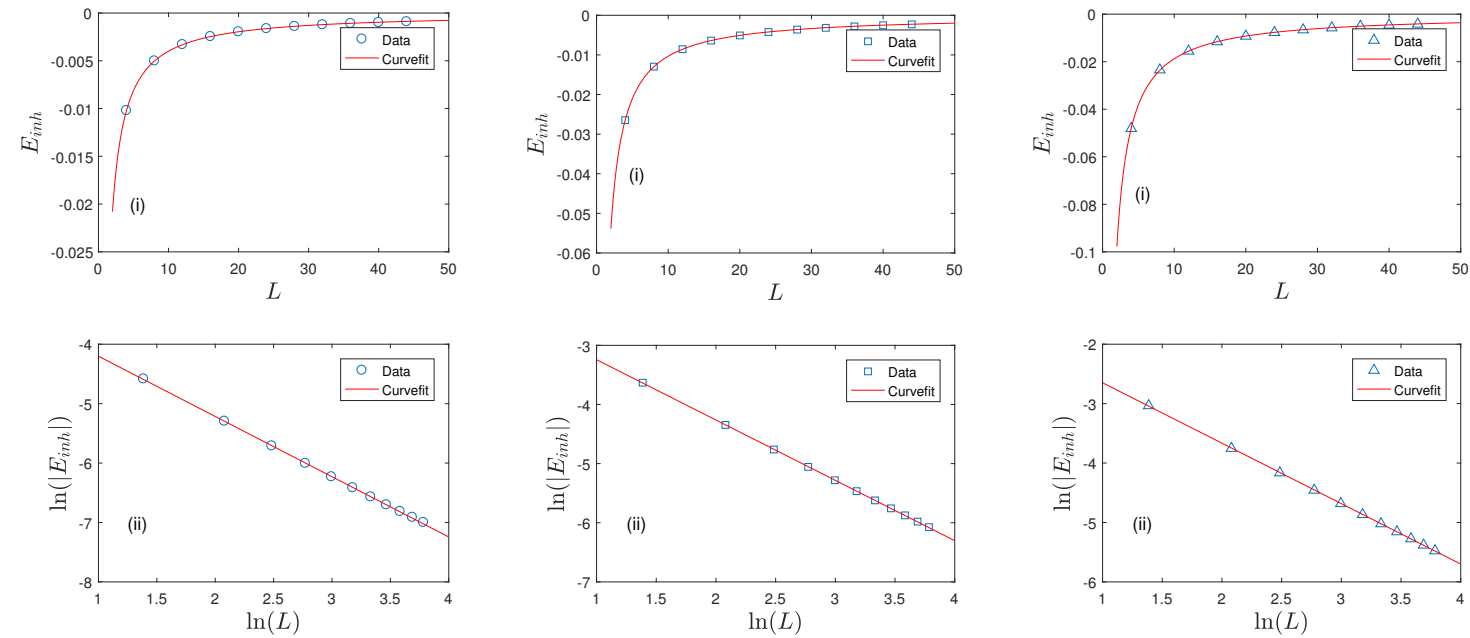

(a) $\theta^{\prime}=0.80$.

(b) $\theta^{\prime}=1.20$.

(c) $\theta^{\prime}=1.57$.

Figure 3. The values of $E_{\text {inh }}$ versus the system size $L$. The data can be fitted as $E_{\text {inh }}=\tilde{\gamma} L^{\tilde{\beta}}$ [or $\left.\ln \left(\left|E_{\mathrm{inh}}\right|\right)=\tilde{p} \ln (L)+\tilde{q}\right]$. Here $\xi=-0.1, \xi^{\prime}=0.95, \theta=0.15, \phi=\phi^{\prime}=0$, (a) $\theta^{\prime}=0.80, \tilde{\gamma}=0.0423$ and $\tilde{\beta}=-1.0250$; (b) $\theta^{\prime}=1.20, \tilde{\gamma}=0.1097$ and $\tilde{\beta}=-1.0260$; (c) $\theta^{\prime}=1.57, \tilde{\gamma}=0.1988$ and $\tilde{\beta}=-1.0250$. Due to the fact $\tilde{\beta}<0$ [or $\tilde{p}<0]$, when the $L$ tends to infinity, the $E_{\mathrm{inh}}$ tends to zero.

where the quantum numbers $\left\{I_{j}\right\}$ are chosen as $\{1,2, \cdots, L / 2-1\}$. The corresponding energy reads

$$
E_{\text {hom }}=-\sum_{k=1}^{M-1} \frac{1}{\mu_{k}^{2}+\frac{1}{4}}-\frac{1}{\frac{1}{4}-\zeta^{2}}+2 M .
$$

The values of $E_{\text {inh }}$ versus the system size $L$ are shown in figure 3 . From the fitted curves in figure 3 , we see that the $E_{\text {inh }}$ also satisfies the scaling law $L^{-1} \cdot{ }^{4}$ Therefore, the contribution of the inhomogeneous term to the ground state energy in the thermodynamic limit is zero and we have $E_{\text {hom }}=E_{\text {ture }}=E$. In addition, the results indicating that the boundary bound state should be stable. The surface energy will compute in the next section.

\section{Surface energy}

In order to analyze the influence of the boundary fields, now we calculate the surface energy [40-42] of the system.

\subsection{Region of $\xi<0$ and $\xi^{\prime} \leq 1 / 2$}

By defining $Z\left(\mu_{j}\right)=\frac{I_{j}}{2 L}$, the BAEs (3.10) can be rewritten as

$$
Z\left(\mu_{j}\right)=\frac{1}{2 \pi}\left[\frac{1}{2 L} \Xi_{2 \zeta}\left(\mu_{j}\right)+\Xi_{1}\left(\mu_{j}\right)\right],
$$

\footnotetext{
${ }^{4}$ It should be emphasized that, for a finite $L$, the causes of the difference $E_{\text {inh }}$ included two aspects, omitting the exponentially small corrections and ignoring the contribution of the inhomogeneous term.
} 


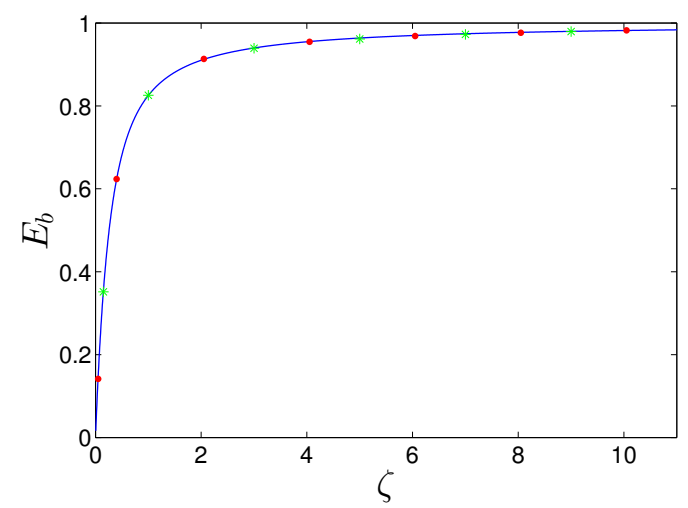

Figure 4. The surface energies versus the boundary parameters. The blue curves are the ones calculated from equation (4.7), while the red points $\left(\xi=-0.1, \theta=\pi / 5, \phi=0, \theta^{\prime}=0\right.$ and $\left.\phi^{\prime}=0\right)$ and green stars $\left(\xi=-5, \theta=\pi / 4, \phi=0, \theta^{\prime}=2 \pi / 3\right.$ and $\left.\phi^{\prime}=0\right)$ are the ones obtained from Hamiltonian (1.1) with the BST algorithms.

where $\Xi_{n}(x)=2 \arctan (2 x / n)$. It turns to be a continuous function in the thermodynamic limit as the distribution of Bethe roots is continuous, i.e., $Z\left(\mu_{j}\right) \rightarrow Z(u)$. In the thermodynamic limit, the density distributions are determined by

$$
\rho(u)+\rho^{h}(u)=\frac{d Z(u)}{d u} .
$$

Taking the derivative of $Z(u)$ with respect to $u$, we obtain the density of states as

$$
\rho(u)=a_{1}(u)+\frac{1}{2 L}\left[a_{2 \zeta}(u)-\delta(u)\right],
$$

where

$$
a_{n}(u)=\frac{1}{2 \pi} \frac{n}{u^{2}+\frac{n^{2}}{4}} .
$$

Then the ground state energy is

$$
E=-2 \pi L \int_{-B}^{B}\left(a_{1}(\mu)\right)^{2} d \mu+2 M-\pi \int_{-B}^{B} a_{1}(\mu)\left[a_{2 \zeta}(\mu)-\delta(\mu)\right] d \mu,
$$

and the energy density of the ground state is

$$
e_{g}=-2 \pi \int_{-B}^{B}\left(a_{1}(\mu)\right)^{2} d \mu+1+O\left(L^{-1}\right)
$$

where $B=\frac{1}{2}+\frac{\pi-2 \arctan \left(\frac{1}{2 \zeta}\right)}{4 L+\frac{4 \zeta}{1+4 \zeta^{2}}}$. The energy density $e_{g}$ is equal to $-2 / \pi$ in the thermodynamic limit, which is the same as that of the periodic case [43]. The surface energy then can be given by

$$
\begin{aligned}
E_{b}(\zeta) & =\lim _{L \rightarrow \infty}\left[E_{0}(L ; \zeta)-E_{0}^{\text {periodic }}(L)\right] \\
& =-\pi \int_{-\frac{1}{2}}^{\frac{1}{2}} a_{1}(\mu) a_{2 \zeta}(\mu) d \mu+\frac{2}{\pi} \arctan \left(\frac{1}{2 \zeta}\right)+1
\end{aligned}
$$




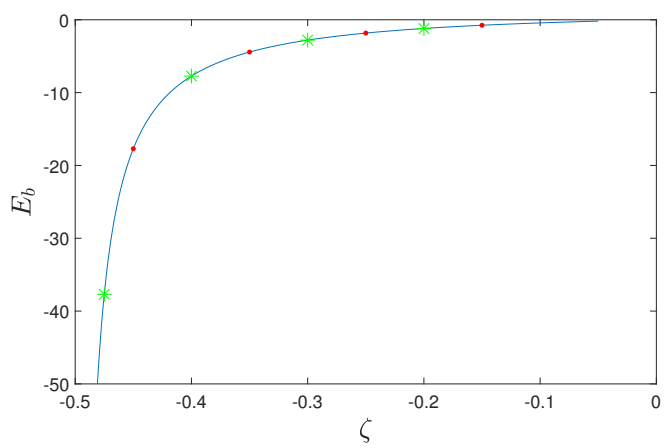

Figure 5. The surface energies versus the boundary parameters. The blue curves are the ones calculated from equation (4.10), while the red points $\left(\xi=-0.1, \theta=0.3 \pi, \phi=0, \theta^{\prime}=0.7 \pi\right.$ and $\left.\phi^{\prime}=0\right)$ and green stars $\left(\xi=-0.1, \theta=0.17 \pi, \phi=0, \theta^{\prime}=0.62 \pi\right.$ and $\left.\phi^{\prime}=0\right)$ are the ones obtained from Hamiltonian (1.1) with the BST algorithms.

By using the relation (4.7), one can calculate the surface energy of the one-dimensional supersymmetric $t-J$ model with unparallel boundary fields. The results are shown in figure 4, where the blue solid lines are the surface energy calculated by using the relation (4.7) and the red points and green stars are data obtained by employing the BST algorithms [44] to solve the surface energy of Hamiltonian (1.1) in the thermodynamic limit. Specifically, for one of the red points or green stars, we first calculate the ground state energy $E_{0}(L)$ with $L=4,8, \cdots, 48$ by the DMRG. Then, the large- $L$ extrapolation of the surface energy was performed using BST algorithms from the sequence $E_{0}(4)-4 e_{g}^{\infty}, E_{0}(8)-8 e_{g}^{\infty}, \cdots$, $E_{0}(48)-48 e_{g}^{\infty}$. Note that $e_{g}^{\infty}=-2 / \pi$. From the figure 4 , we can see that the analytical and numerical results agree with each other very well for all tunable parameters. The surface energy increased with the increase of $\zeta$. Taking the $\zeta \rightarrow 0$ limit of eq. (4.7), we have $E_{b}(\zeta \rightarrow 0)=0$. Taking the $\zeta \rightarrow \infty$ limit of eq. (4.7), we have $E_{b}(\zeta \rightarrow \infty)=1$.

\subsection{Region of $\xi<0$ and $1 / 2<\xi^{\prime}<1$}

In this region, the ground state energy of the system in the thermodynamic limit reads

$$
E=-2 \pi L \int_{-\tilde{B}}^{\tilde{B}}\left(a_{1}(\mu)\right)^{2} d \mu+2 M-\pi \int_{-\tilde{B}}^{\tilde{B}} a_{1}(\mu)\left[a_{2 \zeta}(\mu)-\delta(\mu)\right] d \mu-\frac{1}{\frac{1}{4}-\zeta^{2}},
$$

and the energy density of the ground state is

$$
e_{g}=-2 \pi \int_{-\tilde{B}}^{\tilde{B}}\left(a_{1}(\mu)\right)^{2} d \mu+1+O\left(L^{-1}\right)
$$

where $\tilde{B}=\frac{1}{2}-\frac{\pi+2 \arctan \left(\frac{1}{2 \zeta}\right)}{4 L+\frac{4 \zeta}{1+4 \zeta^{2}}}$. The surface energy is given by

$$
E_{b}(\zeta)=-\pi \int_{-\frac{1}{2}}^{\frac{1}{2}} a_{1}(\mu) a_{2 \zeta}(\mu) d \mu+\frac{2}{\pi} \arctan \left(\frac{1}{2 \zeta}\right)+3-\frac{1}{\frac{1}{4}-\zeta^{2}} .
$$


The results are shown in figure 5. Again, we see that the analytical results and the numerical ones agree with each other very well.

The surface energy can be written in unified forms as

$$
E_{b}(\zeta)=-\pi \int_{-\frac{1}{2}}^{\frac{1}{2}} a_{1}(\mu) a_{2 \zeta}(\mu) d \mu+\frac{2}{\pi} \arctan \left(\frac{1}{2 \zeta}\right)+\Delta,
$$

where $\Delta=1$ when $\xi<0$ and $\xi^{\prime} \leq 1 / 2$, and $\Delta=3-\frac{1}{\frac{1}{4}-\zeta^{2}}$ when $\xi<0$ and $1 / 2<\xi^{\prime}<1$.

\section{Conclusions}

In this paper, we have studied the thermodynamic limit of the one-dimensional supersymmetric $t-J$ model with unparallel boundary fields. It is shown that the contribution of the inhomogeneous term to the ground state energy is inversely proportional with $L$, i.e., $E_{\text {inh }} \propto L^{-1}$. This fact enables us to calculate the surface energy (4.7) and (4.10), which is the same as that for the case of parallel boundary fields [23]. Moreover, it implies that the inhomogeneous term in (2.18) surely gives some contributions to the other physical qualities such as the boundary conformal charge, which are related to the coefficients in the expansion of energy $E$ in terms of the powers of $L^{-1}$ (namely, the coefficient of $L^{-1}$ corresponds to the conformal charge [38]).

The method used in this paper can be generalized to study the thermodynamic limit and surface energy of other models related to rational $R$-matrices, such as the spin- $s$ XXX chain or the $s u(n)$ spin chain with unparallel boundary fields. These results may be applied to the theory of ultra-cold atom systems, asymmetric simple exclusion process.

\section{Acknowledgments}

We would like to thank Prof. Y. Wang for his valuable discussions and continuous encouragements. The financial supports from the National Program for Basic Research of MOST (Grant No. 2016YFA0300600 and 2016YFA0302104), the National Natural Science Foundation of China (Grant Nos. 11434013, 11425522, 11547045, 11774397, 11775178 and 11775177), the Major Basic Research Program of Natural Science of Shaanxi Province (Grant Nos. 2017KCT-12 and 2017ZDJC-32), the Strategic Priority Research Program of the Chinese Academy of Sciences, and the Double First-Class University Construction Project of Northwest University are gratefully acknowledged. F. Wen also acknowledges the support of the NWU graduate student innovation fund (No. YYB17003).

Open Access. This article is distributed under the terms of the Creative Commons Attribution License (CC-BY 4.0), which permits any use, distribution and reproduction in any medium, provided the original author(s) and source are credited.

\section{References}

[1] J. Spalek and A.M. Oles, Ferromagnetism in narrow S-band with inclusion of intersite correlations, Physica B+C 86 (1977) 375. 
[2] K.A. Chao, J. Spalek and A.M. Oles, Kinetic exchange interaction in a narrow S-band, J. Phys. C 10 (1977) L271.

[3] F. Zhang and T. Rice, Effective Hamiltonian for the superconducting Cu oxides, Phys. Rev. B 37 (1988) 3759 [inSPIRE].

[4] P.W. Anderson, The resonating valence bond state in $\mathrm{La}_{2} \mathrm{CuO}_{4}$ and superconductivity, Science 235 (1987) 1196 [inSPIRE].

[5] F.H.L. Essler, V.E. Korepin and K. Schoutens, New exactly solvable model of strongly correlated electrons motivated by high $T_{c}$ superconductivity, Phys. Rev. Lett. 68 (1992) 2960 [cond-mat/9209002] [INSPIRE].

[6] Y.Q. Chong, V. Murg, V.E. Korepin and F. Verstraete, Nested algebraic Bethe ansatz for the supersymmetric $t-J$ model and tensor networks, Phys. Rev. B 91 (2015) 195132.

[7] S. Reja, J.V.D. Brink and S. Nishimoto, Strongly enhanced superconductivity in coupled $t-J$ segments, Phys. Rev. Lett. 116 (2016) 067002.

[8] F.H.L. Essler and V.E. Korepin, Higher conservation laws and algebraic Bethe ansatze for the supersymmetric $t-J$ model, Phys. Rev. B 46 (1992) 9147 [InSPIRE].

[9] H. Fan, M. Wadati and X.-M. Wang, Exact diagonalization of the generalized supersymmetric $t-J$ model with boundaries, Phys. Rev. B 61 (2000) 3450.

[10] W. Galleas, Spectrum of the supersymmetric $t-J$ model with non-diagonal open boundaries, Nucl. Phys. B 777 (2007) 352 [InSPIRE].

[11] E.K. Sklyanin, Boundary conditions for integrable quantum systems, J. Phys. A 21 (1988) 2375 [inSPIRE].

[12] P.B. Wiegmann, Superconductivity in strongly correlated electronic systems and confinement versus deconfinement phenomenon, Phys. Rev. Lett. 60 (1988) 821 [InSPIRE].

[13] D. Forster, Staggered spin and statistics in the supersymmetric $t-J$ model, Phys. Rev. Lett. 63 (1989) 2140.

[14] A. Foerster and M. Karowski, Completeness of the Bethe states for the supersymmetric $t-J$ model, Phys. Rev. B 46 (1992) 9234.

[15] A. Foerster and M. Karowski, Algebraic properties of the Bethe ansatz for an $\operatorname{spl}(2,1)$ supersymmetric $t-J$ model, Nucl. Phys. B 396 (1993) 611 [INSPIRE].

[16] B. Sutherland, A general model for multicomponent quantum systems, Phys. Rev. B 12 (1975) 3795 [INSPIRE].

[17] P. Schlottmann, Integrable narrow-band model with possible relevance to heavy-fermion systems, Phys. Rev. B 36 (1987) 5177 [INSPIRE].

[18] H.J. Schulz, Correlation exponents and the metal-insulator transition in the one-dimensional Hubbard model, Phys. Rev. Lett. 64 (1990) 2831.

[19] H.M. Babujian and R. Flume, Off-shell Bethe ansatz equation for Gaudin magnets and solutions of Knizhnik-Zamolodchikov equations, Mod. Phys. Lett. A 9 (1994) 2029 [hep-th/9310110] [INSPIRE].

[20] H.M. Babujian, A. Foerster and M. Karowski, The nested SU(N) off-shell Bethe ansatz and exact form-factors, J. Phys. A 41 (2008) 275202 [hep-th/0611012] [INSPIRE]. 
[21] H.M. Babujian, A. Foerster and M. Karowski, $O(N)$-matrix difference equations and a nested Bethe ansatz, J. Phys. A 45 (2012) 055207.

[22] A.S. Mishchenko and N. Nagaosa, Electron-phonon coupling and a polaron in the $t-J$ model: from the weak to the strong coupling regime, Phys. Rev. Lett. 93 (2004) 036402.

[23] F.H.L. Essler, The supersymmetric $t-J$ model with a boundary, J. Phys. A 29 (1996) 6183.

[24] J. Sirker and A. Klumper, Thermodynamics and crossover phenomena in the correlation lengths of the one-dimensional $t-J$ model, Phys. Rev. 66 (2002) 245102 [cond-mat/0212437].

[25] M. Takahashi, Thermodynamics of one-dimensional solvable models, Cambridge University Press, Cambridge, U.K., (1999).

[26] Y. Wang, W.-L. Yang, J. Cao and K. Shi, Off-diagonal Bethe ansatz for exactly solvable models, Springer Press, Berlin, Heidelberg, Germany, (2015).

[27] X. Zhang, J. Cao, W.-L. Yang, K. Shi and Y. Wang, Exact solution of the one-dimensional super-symmetric $t-J$ model with unparallel boundary fields, J. Stat. Mech. 04 (2014) P04031 [arXiv:1312.0376] [INSPIRE].

[28] P. Sun et al., On the Bethe states of the one-dimensional supersymmetric $t-J$ model with generic open boundaries, JHEP 07 (2017) 051 [arXiv: 1705. 09478] [INSPIRE].

[29] J. Cao, W.-L. Yang, K. Shi and Y. Wang, Off-diagonal Bethe ansatz and exact solution of a topological spin ring, Phys. Rev. Lett. 111 (2013) 137201 [arXiv:1305.7328] [INSPIRE].

[30] R.I. Nepomechie and C. Wang, Boundary energy of the open XXX chain with a non-diagonal boundary term, J. Phys. A 47 (2014) 032001 [arXiv:1310.6305] [InSPIRE].

[31] Y.-Y. Li, J. Cao, W.-L. Yang, K. Shi and Y. Wang, Thermodynamic limit and surface energy of the XXZ spin chain with arbitrary boundary fields, Nucl. Phys. B 884 (2014) 17 [arXiv: 1401.3045] [INSPIRE].

[32] F. Wen, T. Yang, Z.-Y. Yang, J. Cao, K. Hao and W.-L. Yang, Thermodynamic limit and boundary energy of the su(3) spin chain with non-diagonal boundary fields, Nucl. Phys. B 915 (2017) 119 [arXiv:1608.04634] [inSPIRE].

[33] L. Corwin, Y. Ne'eman and S. Sternberg, Graded Lie algebras in mathematics and physics (Bose-Fermi symmetry), Rev. Mod. Phys. 47 (1975) 573 [InSPIRE].

[34] R.I. Nepomechie, An inhomogeneous $T-Q$ equation for the open XXX chain with general boundary terms: completeness and arbitrary spin, J. Phys. A 46 (2013) 442002 [arXiv:1307.5049] [INSPIRE].

[35] N. Kitanine, J.-M. Maillet and G. Niccoli, Open spin chains with generic integrable boundaries: Baxter equation and Bethe ansatz completeness from separation of variables, J. Stat. Mech. 1405 (2014) P05015 [arXiv:1401.4901] [INSPIRE].

[36] B. Bauer et al., The ALPS project release 2.0: open source software for strongly correlated systems, J. Stat. Mech. 5 (2011) 05001 [arXiv:1101.2646].

[37] S. Skorik and H. Saleur, Boundary bound states and boundary bootstrap in the sine-Gordon model with Dirichlet boundary conditions, J. Phys. A 28 (1995) 6605 [hep-th/9502011] [INSPIRE].

[38] Y. Wang, J. Voit and Fu-Cho Pu, Exact boundary critical exponents and tunneling effects in integrable models for quantum wires, Phys. Rev. B 54 (1996) 8491. 
[39] Y. Wang, Exact solution of the open Heisenberg chain with two impurities, Phys. Rev. B 56 (1997) 14045.

[40] M. Gaudin, Boundary energy of a Bose gas in one dimension, Phys. Rev. A 4 (1971) 386 [INSPIRE].

[41] C.J. Hamer, G.R.W. Quispel and M.T. Batchelor, Conformal anomaly and surface energy for Potts and Ashkin-Teller quantum chains, J. Phys. A 20 (1987) 5677 [InSPIRE].

[42] M.T. Batchelor and C.J. Hamer, Surface energy of integrable quantum spin chains, J. Phys. A 23 (1990) 761 [inSPIRE].

[43] C.K. Lai, Lattice gas with nearest-neighbor interaction in one dimension with arbitrary statistics, J. Math. Phys. 15 (1974) 1675.

[44] M. Henkel and G. Schutz, Finite lattice extrapolation algorithms, J. Phys. A 21 (1988) 2617 [INSPIRE]. 